\title{
Decreasing Stress for Parents of Special Needs Children through a Web-Based Mindfulness Program: A Pilot Study
}

\author{
Elizabeth J. Shaffer \\ Chatham University, elizabeth@therapyintl.com
}

Jennifer E. Lape

Chatham University, jlape@chatham.edu

Joyce Salls

Chatham University, salls@chatham.edu

Follow this and additional works at: https://nsuworks.nova.edu/ijahsp

Part of the Medicine and Health Sciences Commons

\section{Recommended Citation}

Shaffer EJ, Lape JE, Salls J. Decreasing Stress for Parents of Special Needs Children through a WebBased Mindfulness Program: A Pilot Study. The Internet Journal of Allied Health Sciences and Practice. 2020 Jan 01;18(4), Article 16.

This Manuscript is brought to you for free and open access by the College of Health Care Sciences at NSUWorks. It has been accepted for inclusion in Internet Journal of Allied Health Sciences and Practice by an authorized editor of NSUWorks. For more information, please contact nsuworks@nova.edu. 


\title{
Decreasing Stress for Parents of Special Needs Children through a Web-Based Mindfulness Program: A Pilot Study
}

\begin{abstract}
Purpose: Parents often experience moderate to severe levels of stress due to the challenges of raising a child with special needs. Previous research has proven that excessive parental stress negatively impacts both the parent and child's health. However, few organizations have been identified which offer intervention programs focusing directly on decreasing parental stress. This pilot study investigates the effectiveness of a 6-week online mindfulness-based course in reducing stress experienced by parents of children with special needs. Method: A pre-test, post-test pilot design, as well as a participatory action approach, were employed to determine the impact of the online mindfulness-based course in reducing perceived stress experienced by parents of children with special needs. Eleven parents participated in the pilot study. The course was facilitated by an occupational therapist (the first author) trained and experienced in mindfulness practices and a parent of two children with special needs. The Depression Anxiety Stress Scale - Stress subscale (DASS-SS), The Perceived Stress Scale (PSS), The Five Facet Mindfulness Questionnaire-15 (FFMQ-15), and author-generated measures were administered pre- and postintervention to determine changes in perceived stress levels, as well as, the impact and application of mindfulness practices. Results: The DASS-SS measuring the parents' perceived stress level revealed a $30 \%$ mean reduction in stress when comparing pre-and post-intervention scores. The PSS revealed a $21 \%$ mean reduction in perceived stress score post-intervention. The FFMQ-15 (used to measure changes in the integration of mindfulness practices into the parent's daily routine) indicated a $4 \%$ increase in the groups' mindfulness practices comparing pre- and post-intervention scores. Responses to the authorgenerated measure revealed that $82 \%$ of parents believed they could better handle challenging situations post-intervention; $100 \%$ of the parents agreed that the course made them more mindful of their thoughts. Conclusion: Results indicate that an introductory online mindfulness-based parenting program can be an effective intervention for reducing the stress experienced by parents of children with special needs.
\end{abstract}

\section{Author Bio(s)}

Elizabeth J. Shaffer OTD, OTR/L, is a U.S. board-certified occupational therapist. She is also the founder of Therapeutic Innovations International, LLC, an organization that supports families and children around the world.

Jennifer E. Lape, OTD, OTR/L is an Assistant Professor of Occupational Therapy at Chatham University in Pittsburgh, Pennsylvania. She is a licensed occupational therapist in the state of Pennsylvania.

Joyce Salls, OTD, OTR/L, Professor and Director of Occupational Therapy at Chatham University, has 30+ years' experience as an OT practitioner. Dr. Salls has presented nationally and internationally on topics related to pediatrics and educational practices. Her research interests and publications are in areas of program outcomes and school-based services. 


\title{
IIIJAHSP \\ The Internet Joutnal of Allied Health Sciences and Practice \\ Dedicated to allied health professional practice and education
}

Vol. 18 No. 4 ISSN 1540-580X

\section{Decreasing Stress for Parents of Special Needs Children through a Web-Based Mindfulness Program: A Pilot Study}

\author{
Elizabeth J. Shaffer \\ Jennifer E. Lape \\ Joyce Salls \\ Chatham University \\ United States
}

\begin{abstract}
Purpose: Parents often experience moderate to severe levels of stress due to the challenges of raising a child with special needs. Previous research has proven that excessive parental stress negatively impacts both the parent and child's health. However, few organizations have been identified which offer intervention programs focusing directly on decreasing parental stress. This pilot study investigates the effectiveness of a 6-week online mindfulness-based course in reducing the stress experienced by parents of children with special needs. Method: A pre-test, post-test pilot design, and a participatory action approach, were employed to determine the impact of the online mindfulness-based course in reducing perceived stress experienced by parents of children with special needs. Eleven parents participated in the pilot study. The course was facilitated by an occupational therapist (the first author) trained and experienced in mindfulness practices and a parent of two children with special needs. The Depression Anxiety Stress Scale - Stress subscale (DASS-SS), The Perceived Stress Scale (PSS), The Five Facet Mindfulness Questionnaire-15 (FFMQ-15), and author-generated measures were administered pre- and post-intervention to determine changes in perceived stress levels, as well as, the impact and application of mindfulness practices. Results: The DASS-SS measuring the parents' perceived stress level over one week revealed a 30\% mean reduction in stress when comparing pre-and post-intervention scores. The PSS measuring the parents' perceived stress level over one month showed a $21 \%$ mean reduction in perceived stress score post-intervention. The FFMQ-15 (used to measure changes in the integration of mindfulness practices into the parent's daily routine) indicated a $4 \%$ increase in the groups' application of mindfulness practices comparing pre- and post-intervention scores. Responses to the author-generated measure revealed that $82 \%$ of parents believed they could better handle challenging situations post-intervention; $100 \%$ of the parents agreed that the course made them more mindful of their thoughts. Conclusion: Results indicate that an introductory online mindfulness-based parenting program can be an effective intervention for reducing the stress experienced by parents of children with special needs.
\end{abstract}

Keywords: mindfulness, parent education, parental support, special needs, autism, ADHD, stress, well-being, online 


\section{INTRODUCTION}

Parents raising children with special needs are more likely to encounter additional challenges, responsibilities, and emotional and financial burdens than parents of a child without special needs. Many parents report feeling "stretched beyond their limits" and "unable to cope" with the associated challenges of raising a child with autism. ${ }^{1}$ Parents of children with autism spectrum disorder (ASD) are more susceptible to elevated stress, clinical depression, and anxiety disorders. ${ }^{2,3}$ Parents of children with other neurodevelopmental disabilities, including attention deficit hyperactivity disorder (ADHD), are also susceptible to elevated stress levels. ${ }^{4,5}$ The sources of parental stress, as well as coping methods, vary for each family as stress levels are unique to an individual and are impacted by intrinsic and extrinsic factors. Commonly identified sources of stress affecting parents raising children with special needs include financial burdens, the child's aggressive or challenging behaviors, and the uncertainty of the child's potential to overcome obstacles in development. ${ }^{6-8}$ The parent's perceptions of the situation, association of the current stressful situation to past events with adverse outcomes, the present state of physical, mental, and emotional health, and knowledge and ability to practice effective coping strategies also influences the parents perceived stress levels. . $^{8-10}$ Use of blaming, escaping, and avoidance as coping strategies may result in additional adverse outcomes. ${ }^{8}$

Excessive stress experienced by parents and other primary caregivers of children with developmental disabilities is pervasive and is linked to a lower quality of life, unhealthy family functioning, and adverse psychological consequences. ${ }^{11}$ Advancements in the medical community's understanding of the implications of parental stress on both the parent and child's health and well-being is emerging. 2,9,11-13 However, current therapeutic and medical approaches tend to focus on the child's health and well-being exclusively and often overlook the challenges parents face and their ability to deal with the special needs of their child successfully. The parent's state of health and well-being can have a direct impact on the child's welfare. A highly stressed parent is less effective in his or her parenting role, which may limit or adversely impact a child's developmental progress and reduce the effectiveness of early interventions. ${ }^{14,15}$ Children with special needs often require sustained attention, patience, resilience, and determination from the adult to stimulate the child to engage in self-care and play-based activities. A stressed and overwhelmed parent is less likely to have the patience required to engage with their special needs child and implement recommended therapeutic activities. ${ }^{14}$ In addition, excessive stress experienced by the parent can negatively impact relations between the parent and child. Correlations between the mothers' increased stress levels and conflicts between the mother and child have been identified. ${ }^{7}$ Studies have also indicated that parents under excessive stress may react to the negative behaviors of their child with ADHD in a manner that can exacerbate the clinical symptoms of the child with an ADHD disorder. ${ }^{4}$ Lastly, the emotional reactions, behaviors, and symptoms of children with ASD are perceived by parents to be influenced by the parent's emotional state. ${ }^{16}$ Research suggests that helping parents with emotional regulation is a good initial intervention for families of children with special needs. ${ }^{16}$

This pilot study emerged from the understanding that parents of children with special needs endure higher than normal levels of stress and the perceived need to provide parents with the knowledge and tools to manage stress effectively. A review of current literature reveals a wealth of evidence indicating mindfulness-based programs are effective in decreasing stress and increasing emotional regulation in parents of children with various diagnoses. . $^{311,17-20}$ Following this evidence, the first author devised a sixweek mindfulness-based course for parents of children with special needs. Understanding the lack of community support available to some parents due to their geographical location, the first author chose a web-based intervention to allow access to all interested participants regardless of their physical location. Research on the effectiveness of an interactive mindfulness-based intervention for parents of children with special needs using an online platform had not occurred at the time of implementation.

Providing parents with the tools to effectively manage stress and respond to challenging situations can benefit both parents and their children. Research has shown that the mindfulness-based stress reduction (MBSR) program developed by Jon Kabat-Zinn, Ph.D. is an effective program for reducing stress in parents of children with special needs. ${ }^{11}$ In addition, parents who have participated in a mindfulness-based program have demonstrated significant reductions in anxiety and stress and improvements in empathic concern, which can foster positive parent-child relations. ${ }^{10,20}$ Mindfulness-based interventions are comprised of activities to increase the persons' ability to be attentive to the present moment, and often include meditation practices as well as activities that promote self-awareness. Evidence has shown that practicing mindfulness results in better emotional self-regulation during periods of acute stress and increases cognitive flexibility. ${ }^{19,21}$ Therefore, the caregiver who practices mindfulness may be more present and aware of their reactions in the moment and may respond without negative emotional or perceptual influences. Potential benefits may also extend to the child. Parents who participated in mindfulness-based programs noted reduced behavior problems and increased social skills in their children.,5,18,22 When the parent practices self-compassion, they may approach stressful moments with more presence and ease, which may provide the child with a more positive model for responding to challenging situations. ${ }^{23}$ While few identified organizations offer programs to parents that specifically addresses parental stress, existing research has validated the numerous benefits experienced by parents of children with special needs upon completing a mindfulness-based stress reduction program. $3,11,17-20$ 
This pilot study followed a participatory action approach considering the challenges and ambitions of study participants. ${ }^{24}$ The intervention and study design considers' the parents' unique challenges and ambitions raising children with special needs. The first author, an experienced occupational therapist and mother of two children with autism, selected the mindfulness-based elements to be included within the course based on clinical and intimate observations of the population's perceived needs and challenges. To ensure the group's distinct needs and challenges are recognized, parents were asked during the first class to identify their main challenge or source of stress experienced as a parent raising a child with special needs. Throughout the course, their responses were considered when presenting mindfulness-based practices that could be applied when faced with the identified challenges. Participatory action research aims to improve health and reduce health inequities by involving the people who will be taking action to improve their health. ${ }^{24}$ Classes were structured to facilitate an interactive environment in which parents take an active role in the group by participating in open discussions. After introducing a mindfulness-based practice, the group discusses how it relates to their identified stressful and challenging experiences. To further ensure the intervention recognizes the population's desires, parents had the opportunity during the post-intervention survey to share their thoughts and opinions regarding course content and offer suggestions for improvements to be considered in future programs. Each class contained fundamental mindfulness-based practices to ensure the intervention under study followed similar teachings applied in previous studies and to prevent potential bias in the design of the intervention. $20,25,26$

\section{METHODS}

Participants

Recruitment involved posting a recruitment flyer in two members-only social media groups for parents of children with special needs affiliated with the U.S. Foreign Service. The first author is a Foreign Service family member and is familiar with this population's increased potential for experiencing additional stresses resulting from frequent moves, adaptations to living in new cultures, and changes in regulations supporting families with special needs. The Institutional Review Board at Chatham University approved the pilot study. Twelve parents contacted the first author through the email posted on the recruitment flyer. Each parent was then provided with the informed consent and intake questionnaire and offered the opportunity to ask questions via email or phone. Each parent completed, signed, and returned the required forms electronically. Parents' responses to the intake questions were reviewed to ensure each met inclusion criteria. All interested parents met criterion with one lost due to attrition prior to the first class. Criteria for inclusion in this pilot study included: biological or adoptive parent of a child with special needs, fluency in the English language, and access to the necessary technology (computer, tablet, or smartphone, with a camera, microphone, and internet connection). For this study, a child was considered to have special needs if he or she required additional support due to a cognitive, emotional, or physical disability, as reported by the parent or guardian. Eleven parents participated in the study. While recruitment targeted both mothers and fathers, only mothers expressed an interest in joining the study. All participants were American citizens residing in the following countries: North America (5), South America (2), Europe (1), and Africa (3). Table 1 further summarizes the demographic profiles of each participant.

Table 1. Participant Demographic Profiles ( $\mathrm{N}=11)$

\begin{tabular}{llcc}
\hline Sample characteristics & & $\mathbf{N}$ & $\%$ \\
\hline Gender & Female & 11 & 100 \\
& Male & 0 & 0 \\
Marital Status & Married & 10 & 91 \\
& Divorced/Separated & 1 & 9 \\
Parent's Age Range & $20-29$ & 0 & 0 \\
(In years) & $30-39$ & 4 & 36 \\
& $40-49$ & 5 & 46 \\
& $50-59$ & 2 & 18 \\
Number of Children & 1 & 2 & 18 \\
& 2 & 8 & 73 \\
Number of Children in the family with & 3 & 1 & 9 \\
Special Needs & 1 & 7 & 64 \\
& 2 & 4 & 36 \\
Age of Children (In years) & $1-3$ & 3 & 14
\end{tabular}


(With and without special needs)

Diagnosis or Identified Delay in

Development as Reported by Parent (Some

children had more than one identified

diagnosis.) $\begin{array}{lll}4-6 & 2 & 10 \\ 7-9 & 6 & 29 \\ 10-12 & 4 & 19 \\ 13-15 & 3 & 14 \\ 16-18 & 3 & 14\end{array}$

$\begin{array}{lcc}\text { ADHD/ADD } & 10 & 67 \\ \text { Autism } & 5 & 33 \\ \text { Anxiety } & 5 & 33 \\ \text { Dyspraxia or dysgraphia } & 4 & 27 \\ \text { Dyslexia } & 2 & 13 \\ \text { Speech related disorder } & 3 & 20 \\ \text { Fragile-X syndrome } & 1 & 7\end{array}$

\section{Intervention}

The intervention consisted of six synchronous live and interactive weekly online classes. The parents were introduced to specific mindfulness-based attitudes, engaged in topic-related discussions, and participated in formal mindfulness practices such as breathing exercises and short guided meditations. The course content was created following the mindfulness-based principles, theories, and practices explained by Jon Kabat-Zinn, Bob Stahl, Elisha Goldstein, and Kristen Neff..23,25,26 The six classes were organized sequentially to build upon the parents' understanding of stress, self-regulation, mindfulness-based stress management techniques, and mindfulness-based attitudes and practices. To accommodate different time zones and schedules, participants were offered the choice of participating in either the evening or afternoon class with each class following the same course outline. Three parents chose to participate in the evening class, and nine parents chose the afternoon class. The first 10 minutes of each class was spent reviewing the topic presented during the previous week's class; parents were then invited to share with the group their experiences in integrating mindfulness-based activities into their daily routines. Next, the current week's mindfulness-based topic was introduced, discussed, and practiced as a group. Finally, the topics presented during class were reviewed, and time was allotted to answer questions. Parents were encouraged to practice the activities at home that were discussed during each class. Table 2 includes a summation of content covered each week.

Table 1. Course Topics and Class Content

\begin{tabular}{cll}
\hline Week & Topic & Content \\
\hline 1 & $\begin{array}{l}\text { Introduction to Mindfulness } \\
\text { and Parental Stress }\end{array}$ & $\begin{array}{l}\text { Introductions and class rules; time allotted to complete the PSS, DASS-SS, } \\
\text { FFMQ-15; identify primary stressors and challenges; identify how stress is } \\
\text { experienced; group discussion on the impact of stress within family; } \\
\text { introduction to mindfulness; mind-body-break: S. T.O.P. approach. }\end{array}$ \\
\hline 2 & $\begin{array}{l}\text { Self-regulation and } \\
\text { Responding to Stressful } \\
\text { Situations }\end{array}$ & $\begin{array}{l}\text { Parents identify how they react to stressful events then examine how } \\
\text { internal and external circumstances can impact their responses; identify and } \\
\text { discuss parent's and child's self-regulation strategies; identify states of } \\
\text { arousal and the benefits of allowing time for restoration; mind-body-break: } \\
\text { engage in the mindfulness practices of mindful breathing and stretching. }\end{array}$ \\
\hline 3 & $\begin{array}{l}\text { Being Mindful of Your } \\
\text { Thoughts }\end{array}$ & $\begin{array}{l}\text { Identify common autonomic negative thoughts related to the parent's } \\
\text { identified stressors; group discussion - common thoughts and reactions } \\
\text { during challenging moments; introduce the mindfulness attitude of non- } \\
\text { judgment, mind-body-break: 10-minute guided meditation. }\end{array}$ \\
\hline 4 & $\begin{array}{l}\text { Parenting with a New } \\
\text { Perspective }\end{array}$ & $\begin{array}{l}\text { Examine ways to overcome potential barriers to practicing mindfulness at } \\
\text { home; discuss the impact of self-criticism; introduction into self-compassion; } \\
\text { mind-body-break: love and kindness guided meditation. }\end{array}$ \\
\hline 5 & The Art of Being in the & $\begin{array}{l}\text { Introduction to yoga practices, mindful eating, the mindfulness attitude } \\
\text { "beginners' mind"; Examine how to increase awareness to the present } \\
\text { moment by acknowledging thoughts, sensations, and surroundings; mind- } \\
\text { body-break: body scan meditation. }\end{array}$ \\
\hline
\end{tabular}




\begin{tabular}{cll}
\hline 6 & Incorporating Mindfulness & Review course content, identity, and create a plan to continue mindfulness \\
Practices & & $\begin{array}{l}\text { practices; additional resources provided for continued practice; group } \\
\text { perspectives of mindfulness-based practices; mind-body-break: } \\
\text { concentration through focused breathing. }\end{array}$ \\
\hline & $\begin{array}{l}\text { Email was sent to each parent with a link to complete outcome measures } \\
\text { (PSS, DASS-SS, FFMQ-15) and a 1-page summary of mindfulness topics } \\
\text { covered during the course. }\end{array}$ \\
\hline
\end{tabular}

\section{Outcome Measures}

The stress sub-scale of the Depression Anxiety and Stress Scales (DASS-SS), The Perceived Stress Scale (PSS), and The Five Facet Mindfulness Questionnaire-15 item (FFMQ-15) were utilized in this study to measure the participants' perceived stress levels and integration of mindfulness practices into daily routines. ${ }^{27,28,29}$ The DASS-SS, PSS, and FFMQ-15 were acquired through the public domain and administered pre- and post-intervention using a web-based survey platform. All participants completed the online pre- and post-surveys. Participants were asked to choose a 4-digit code unknown to the facilitator when completing the surveys to maintain confidentiality while permitting individual pre-and post-responses to be compared. Further details on each measure are outlined next.

\section{Depression Anxiety and Stress Scales - Stress subscale}

The Psychology Foundation of Australia's Depression Anxiety and Stress Scales includes three scales measuring depression, anxiety, and stress. As the focus of this study is to identify changes in parental stress levels, only the stress sub-scale was utilized. The Depression Anxiety and Stress Scales - Stress subscale (DASS-SS) is comprised of 10 items assessing the following characteristics associated with stress; difficulty relaxing, nervous arousal, being easily upset/agitated, irritable/overreactive, and impatience. ${ }^{27}$ Participants used a 4-point severity/frequency scale to rate the extent to which they have experienced each state over the past week. The DASS-SS was chosen due to commonalities in measured items and parents' expressed states reported to the first author during clinical experiences with similar populations. The DASS subscales can be completed and scored separately without compromising the validity of the results. Omitting a scale will not have a noticeable effect on scores for the remaining scales ${ }^{27}$ Research has proven the reliability of independently administering the DASS stress - subscale (DASS-SS) in measuring the correlating mood (Cronbach's alpha 0.94). ${ }^{30}$ Research has shown the DASS to have acceptable reliability and convergent validity with other measures investigating depression, anxiety, and stress in adults in nonclinical settings. ${ }^{30}$ Studies have shown the Perceived Stress Scale (PSS) score exhibited high correlation coefficients with DASS-21 subscales scores, stress $(r=0.64)$, therefore, the PSS was added as an additional measure to verify changes in the parents' perceived stress pre- and post-intervention. ${ }^{31}$

\section{Perceived Stress Scale}

The Perceived Stress Scale (PSS) by Sheldon Cohen is a 10-item Likert self-report scale that measures a person's perception of stress focusing on the degree to which situations in one's life are apprised as stressful. ${ }^{28}$ The PSS has been used in previous studies investigating the impact of mindfulness-based interventions on parental stress levels.11,20 The PSS has shown to be a reliable and valid measure when used to identify a person's perceived stress level. ${ }^{31}$ Participants rate how often the indicated feeling and thought within a specified situation was experienced during the last month using a 5-point Likert scale. For example, the person rates, on a scale from 0 (never) to 4 (very often), how often in the last month they have found that they could not cope with all the things they had to do. ${ }^{28}$

\section{The Five Facet Mindfulness Questionnaire - 15}

The Five Facet Mindfulness Questionnaire - 15 (FFMQ-15) measures the application of select mindfulness-based practices and attitudes. The FFMQ-15 is a self-report questionnaire used pre- and post-intervention to investigate potential changes in the parents' inner experiences in the following five mindfulness facets: Observing, Describing, Acting with Awareness, Non-Judging of Inner Experience, and Non-Reactivity. ${ }^{29}$ For example, the parent is asked to determine the accuracy and frequency of the following statement: "I don't pay attention to what I'm doing because I'm daydreaming, worrying, or otherwise distracted."29 Various versions of the FFMQ (including 39-item and 24-item measure) have been used in other studies with similar populations before and after mindfulness-based intervention with comparable results. ${ }^{10,20,32}$ Current research supports the use of the FFMQ15 as a reliable and valid measure for use with pre- and post-intervention studies employing mindfulness-based practices. ${ }^{33}$

\section{Author-generated measure}

Author-generated questions included in the pilot study were implemented to gain a more in-depth perspective into each parent's experience and were exploratory in nature. During the first class, parents were asked to share an example of a primary challenge or stressor faced as a parent of a child with special needs. Parents typed their responses in the private chat box. These 
responses guided related discussions throughout the course, connecting the use of mindfulness practices as a coping and preventative measure to be used with the identified challenges. Additional quantitative and qualitative questions were included in the post-survey to identify the following: changes in the parents' level of confidence for handling challenging situations, changes in parent-child relations, anticipated use of a particular mindfulness-based practice in the future, beneficial elements of the online mindfulness-based intervention, and recommendations for future courses. Questions were devised specifically for this pilot study to gain further insight into the participants' perspectives. These questions were reviewed by two experts in survey design to confirm content validity. Author-generated questions and analyzed responses are outlined in Table 3.

\section{Data Analysis}

The DASS-SS, PSS, and FFMQ-15 were analyzed using mean and percentages to compare changes in parents' perceived stress levels and application of mindfulness practices before and after the 6-week intervention. The population t-test was employed to measure the significance of changes between the group's mean pre-and post-survey scores. Quantitative components of the author-generated survey were analyzed using descriptive statistics. This information was not used to compare changes in a specific area but to acquire feedback from the parents in a particular area. Data obtained from these outcome measures were analyzed using advanced data exports (SSPS) Microsoft $\odot$ Excel for Mac software version 16.20. Qualitative data was obtained through structured, open-ended questions asked within the course and within the post-survey questionnaire. The qualitative statements were analyzed using a deductive approach to identify themes related to the source of parents' perceived stress and parents' perception of the course using a compare and contrast approach. ${ }^{34} \mathrm{~A}$ deductive approach was used as the primary author had theorized potential sources of parental stress drawing from her clinical and personal experiences. Several pre-identified themes were confirmed, and new themes emerged when analyzing parents' responses. The first author identified qualitative themes. These themes were then reviewed and verified by the second and third authors.

\section{RESULTS}

\section{Depression Anxiety Stress Scale - Stress Subscale (DASS-SS)}

The DASS-SS pre-survey results indicated that $82 \%$ of participants experienced moderate to extremely severe stress levels at the start of the study. Comparisons made between the DASS-SS pre and post-survey results reveal that all parents identified as experiencing moderate to extreme stress pre-intervention reported decreased stress levels post-intervention. DASS-SS pre- and post-intervention scores also reveals that $82 \%$ of all parents presented with decreased stress levels post-intervention. The group mean stress score declined from 21.7 to 15.1 post-intervention resulting in a $30.4 \%$ decrease in the group's total stress postintervention. However, this difference did not meet the criterion for statistical significance $(p=0.064)$. A comparison of DASS-SS scores for each parent before and after the intervention is illustrated in Figure 1.

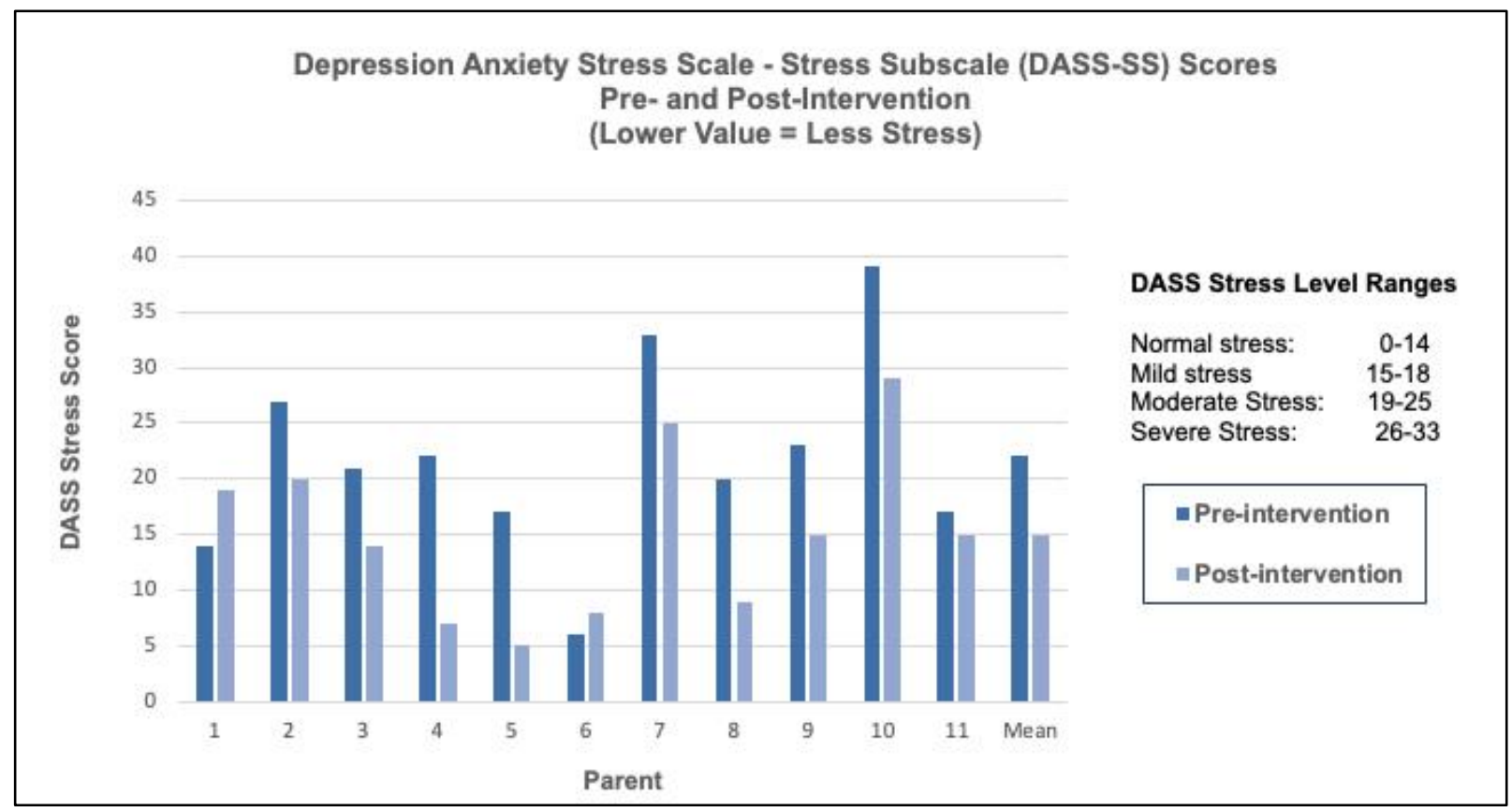

Figure 1. Depression Anxiety Stress Scale Parent Stress Score Pre- and Post-Intervention 


\section{Perceived Stress Scale (PSS)}

The Perceived Stress Scale (PSS) pre-survey results identified that $91 \%$ of the parents in this study experienced moderate to high stress levels at the start of this project. A comparison between the PSS pre- and post-intervention scores reveal that $73 \%$ of the parents presented with decreased stress levels post-intervention. One parent exhibited no changes in perceived stress, and two parents showed a slight increase $(+1)$ in stress levels post-intervention. Analysis of the mean group scores indicates the group's perceived stress levels decreased $21.6 \%$ from an average score of 25.5 to 20.0 post-intervention. Statistically significant changes in the PSS pre- and post-survey scores were identified ( $p=0.053$ ). Figure 2 compares individual pre- and postintervention PSS scores.

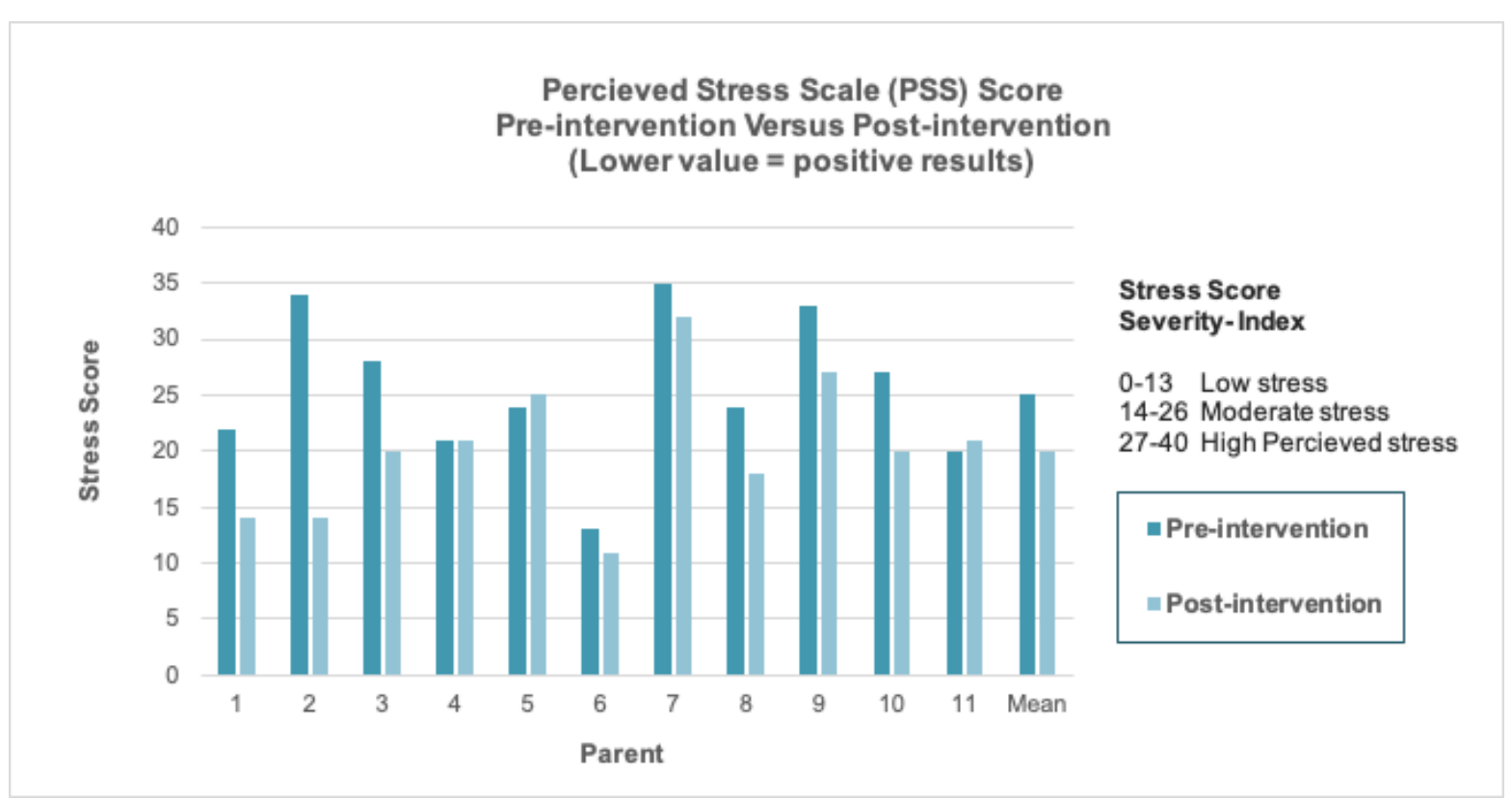

Figure 2. Perceived Stress Scale Score Pre-Intervention Versus Post-intervention

\section{Five Facet Mindfulness Questionnaire - 15 Item (FFMQ-15)}

The FFMQ-15 measured the degree of parents' integration of mindfulness practices into their daily lives. Parents rated how true or how often a statement correlating to a specific mindfulness-based practice or attitude applies. The group's overall mindfulness score following the 6-week introductory mindfulness class increased from 47.3 to 49.4 , indicating a $4.42 \%$ increase in the parents' application of mindfulness practices and attitudes post-intervention. Scores do not reflect a significant change in the group's FFMQ-15 pre- and post-survey scores $(p=0.89)$.

\section{Author Generated Measure - Quantitative Results}

The following author generated questions included in the post-survey aim to identify the frequency that participants meditated at home, which practices parents anticipated continuing after the course, determine the impact of course content on the parent's ability to handle challenging situations, and changes in parent-child relations. Parents selected their answer from the corresponding 5-point Likert scale. Percentage analysis was applied to examine responses (Table 3).

Table 2. Participant Response to Author-Generated Post-Survey Questions

Question
you meditate?
As a result of this class, I am more mindful of
my thoughts.

Percentage of parents selecting the chosen response, $(\mathrm{N}=11)$

$9 \%$ (None)

$46 \%$ (1-5 times)

$36 \%$ (6-10 times)

$9 \%(11-15$ times)

$0 \%$ (16 or more times)

$9 \%$ (Strongly agree)

$91 \%$ (Agree)

$0 \%$ (Neither agree or disagree/Disagree/Strongly disagree) 
I feel stronger in my ability to handle challenging situations than I did six weeks ago.

Relations between my child and I have improved over the past six weeks.
$18 \%$ (Strongly agree)

64\% (Agree)

$18 \%$ (Neither agree or disagree)

0\% (Disagree/Strongly disagree)

9\% (Strongly agree)

$36 \%$ (Agree)

$46 \%$ (Neither agree or disagree)

$9 \%$ (Disagree)

$0 \%$ (Strongly disagree)

\begin{tabular}{l|l}
\hline Indicate which mindfulness practices you would consider contin \\
\begin{tabular}{l|l} 
S.T.O.P. & $63.64 \%(7)$ \\
Guided meditations & $72.73 \%(8)$ \\
Breathing exercises & $72.73 \%(8)$ \\
Yoga & $63.64 \%(7)$ \\
Staying present in the moment & $72.73 \%(8)$ \\
Self-compassion & $72.73 \%(8)$ \\
Acknowledging your thoughts & $63.64 \%(7)$
\end{tabular}
\end{tabular}

\section{Author Generated Measure - Qualitative Results}

This pilot study incorporates a qualitative component for two purposes. The first was to understand better the collective experiences parents identify as their source of stress, and secondly, to identify elements of the course parents found useful. Data was obtained through structured, open-ended questions asked within the course and included in the post-survey questionnaire. The written responses were analyzed using a deductive approach to identify themes. ${ }^{34}$ Several themes/commonalities were noted in the parents' responses to questions posed during the course and within the author generated measure that are worth noting. These themes provide a better understanding of the challenges and obstacles parents of children with special needs experience.

\section{Contributing Factors of Parental Stresses}

During the first class, parents were asked to identify the primary challenge or stressor they faced as a parent of a child with special needs. Parents typed their responses in a private chat box visible only to the first author who recorded these responses. Throughout the course, if a parent shared a specific example of an event that caused them to feel stressed, their responses were written down. These responses were analyzed to identify common themes and contributing factors to increased parental stress (Table 4).

Table 4. Qualitative Themes Related to Contributing Factors of Parental Stress

Themes
Theme 1
Contrasting perspectives regarding child's needs and
proposed solutions.

Theme 2

Parents' thoughts and emotions associated with worries about current and future situations

$\underline{\text { Theme } 3}$

Child's behaviors and special needs

\section{Supporting Quotes}

"I think my child needs more therapy, but my husband disagrees."

"The school wants me to medicate my child, so he will not be disruptive in class." (The parent did not agree with this approach)

"I am so afraid our next school will do the same." [ask us not to return (private international school)] "When my daughter acts bad around her friends, I worry that she will never have close friends as no one will want to play with her."

"My son can't focus on what he needs to, and I worry that he will never be able to hold a job or live on his own."

"None of my children listen to me, and then sometimes they get aggressive towards each other." 


\section{Theme 4}

Perceived judgment from others

\section{Theme 5}

Educational barriers

Theme 6

Parent is not engaging in self-care or restorative activities
"I can't get my child motivated to do his homework or anything for that matter. This is a constant source of frustration in our household."

"My child's emotional needs can be overwhelming at times. Sometimes I just don't have it in me to give him the patience, attention, and compassion he needs when he is having an emotional breakdown."

"I hate the judgment I get from my in-laws who think my child (with autism) is just being bad, and I am a bad mother because my son won't listen to me and stop these behaviors."

"My daughter had a playdate today and acted very badly. I can't imagine what the child's mom thinks of me."

"I hate dealing with new teachers that do not understand how my child learns."

"The last school, my son, was at asked us not to return" "It is so hard finding the right school and services for my son."

"I can't believe after 50 years I have finally realized that it is ok to stop and breathe."

"It is hard to find time for myself."

"I never thought of being compassionate to myself."

"The analogy of putting your own oxygen mask on first is a good one, reminds you that you need to take care of yourself in order to be able to take care of those who depend on you."

"My boys have so many needs that I often forget about my own."

\section{Parents' Perception of the Intervention}

Parents were given the option in the post-survey to share one or two sentences about the course that they believe would be useful to share with parents in the future. Sixty-three percent of the participants responded to the proposed question in the postsurvey. Using this feedback, aspects of the course parents found to be beneficial were identified. Similarities in content and underlying contexts of each response were interpreted and analyzed using a summative approach. Grouping responses identified several themes (Table 5).

Table 5. Emerging Themes Related to the Identified Beneficial Elements of an Online Mindfulness-based Course for Parents of Children with Special Needs.

\begin{tabular}{ll}
\hline Themes & Supporting Quotes \\
\hline Theme 1 & "The class gave me a sense of community, and I felt \\
connected with parents across the world who have similar \\
challenges as I do. That was very powerful for me." \\
"Very helpful. Made me feel less alone." \\
"The group aspect with the exchange of stories and the \\
supportive atmosphere has been an anchor point for me. I \\
wish that could continue."
\end{tabular}

\section{Theme 2}

Awareness of the need for self-care
"The analogy of putting your own oxygen mask on first is a good one, ... you need to take care of yourself in order to be able to take care of those who depend on you." 
"Selfcare is incredibly important for parents of special needs kids."

Theme 3

Mindfulness-based practices and attitudes
"(The course) helps you become more aware of your own actions, it confirms that we often struggle with the same things and gives you some tools to work on what you need as a parent/adult."

"Great resources."

"This class will give you an array of excellent, simple, practical tools to meaningfully improve your selfcare." "I did really enjoy the various elements you brought out in terms of mindfulness - some I already heard of, and some were newer to me, but all very useful"

\section{DISCUSSION}

The pilot study's outcomes are consistent with the current literature showing that parents demonstrate a decrease in perceived stress levels following a mindfulness-based intervention.,14,11,18,20 While mindfulness-based instruction provided to parents in prior studies were conducted in a face-to-face or a facility setting, this pilot study yielded similar results with the use of an interactive web-based platform. These results indicate that a web-based platform may be a viable solution to supporting parents using virtual methods allowing more parents access to this type of support regardless of their geographical location.

\section{Comparing Stress Measure Outcomes}

The DASS-SS and PSS were used to investigate changes in parents' perceived stress levels pre- and post-intervention. All parents in this study experienced decreased stress in at least one measure. Fifty-four percent of parents experienced decreased stress scores post-intervention in both measures. Both measures rate the frequency of each participant's experiences, but the duration of these experiences varies significantly between the two measures. Discrepancies in the individual's responses and significance of change between the two stress measures are likely related to the variances in temporal context within each measure. The DASS-SS asks participants how much the statement applied to them over the past week, whereas the PSS asks participants to rate how often specific thoughts or feelings were experienced within the past month. Short-term metrics will typically show a higher statistical variation among the metrics versus long-term standardization of the data. The DASS-SS measures change over a shorter duration. Temporal differences may account for the higher statistical variations, which may impact the significance of change calculated within this pilot study. Both measures indicate participants in this study experienced a decrease in stress, and any reduction of parental stress is clinically significant.

\section{Stress Related to Child Diagnosis}

The majority of the parents in this pilot study reported moderate to severe stress levels, as revealed in the DASS-SS and PSS pre-intervention stress scores. Recruited parent participants were to have a child with special needs, and no particular diagnosis was targeted. It is worth noting that all of the parents who volunteered to be in the study are raising a child with a neurodevelopmental disorder. The majority of research investigating parental stress reviewed in the preparation of this study focuses on parents of children with neurological impairments. These findings suggest that parents of children with neurological impairments impacting the child's social, emotional, and behavioral abilities experience higher rates of perceived stress. The most common child diagnoses identified by parents within this study were ADHD/ADD (10) and ASD (5). Increased parental stress in this population may also relate to the comorbid mental health disorders associated with neurological conditions. Anxiety, conduct problems, hyperactivity, and oppositional behaviors can be particularly challenging for the parent. Especially if the parent does not have the support or knowledge of how to handle such challenges. ${ }^{35}$ These findings suggest that parents raising children with neurodevelopmental disorders such as autism and ADHD/ADD may need more support than parents of children with other developmental disabilities.

\section{Limitations}

There are three identified limitations to this pilot study. When drawing conclusions from the data, the small sample size and lack of a control group must be considered. Future research examining the impact of online mindfulness-based interventions for parents with special needs should include a larger, more diverse population, and employ a wait-list control group. Second, due to time limits imposed within this pilot study, data identifying long-term changes in parents' level of stress and the longevity of the application of mindfulness practices was not obtained. Thus, future studies should be conducted over an extended period to allow data collection after the intervention to see if parents continued to follow the practices provided throughout the course and to examine if mean stress levels continue to decrease, increase, or remain the same. Lastly, the outcome measures used in this 
study were all self-report measures, which may impact results. A parent's current emotional state of being may directly affect how they consider their feelings were over the duration specified by each stress measure (i.e., over the last week, over the last month). However, to mitigate this limitation, two separate stress measures (DASS-SS and PSS) were chosen to increase the reliability of outcomes. But as these measures ask the participant to identify perceived stress over two different intervals, true comparability between the two outcome measures was not achieved.

\section{RECOMMENDATION AND CONCLUSIONS}

The majority of research examining mindfulness-based interventions for parents of children with special needs follows the Mindfulness-Based Stress Reduction (MBSR) guidelines created by Dr. Jon Kabat-Zinn. ${ }^{11,18,22}$ Additional studies are emerging, including this pilot study, which employs formal and informal mindfulness practices based upon the work of Dr. Kabat-Zinn while specifically adapting this content to address the identified needs of this population. $5,20,32$ Applying mindfulness practices to the participant's shared challenges is not included in the MBSR program. Research shows that effective parent-focused interventions share common features. They provide parents the opportunity to tell their stories, strengthen family relationships by enhancing communication skills, introduce stress and behavior management strategies, and promote problem-solving abilities. ${ }^{37}$ In addition, effective interventions support normalization of the parent-child interaction by acknowledging the specific challenges associated with a child's condition. ${ }^{36}$ Each of these elements were integrated when possible into the design and implementation of this mindfulness-based online course and should be considered when facilitating future mindfulness-based programs for parents.

The mindfulness-based intervention was adjusted to allow parents time to engage in open-dialogues with the facilitator and each other to promote a sense of partnership and belonging among the parents working towards a shared goal. The importance of self-care was acknowledged, and parents explored mindfulness practices that promote stress reduction and management of emotional reactions. One class was devoted to the practice of self-compassion, following the work of Dr. Kristen Neff. Her research suggests that self-compassion may play a significant role in promoting the well-being of parents of children with autism. ${ }^{23}$ Adding the component of self-compassion may provide the emotional support that many parents of children with special needs are lacking. Feedback indicated teachings related to self-compassion were useful and should be considered in future program designs.

Another element worth investigating in future studies is the effectiveness of a web-based platform. The use of an interactive webbased platform within this study allowed parents from various locations worldwide to connect and grow with other parents who are facing similar challenges. Conducting the mindfulness-based intervention online removes demographic barriers permitting more parents to receive this type of support. Search results found no studies investigating the impact of a mindfulness-based program for parents of children with special needs conducted using an interactive web-based platform. Telehealth is becoming a large part of the healthcare landscape as an estimated 15 million Americans received medical care remotely in 2015. ${ }^{37}$ Accessibility of services is a primary factor in the parent's decision whether to participate in parent-based programs. ${ }^{38}$ The trend for more sustainable and accessible health-related interventions requires health and community-based providers to consider moving towards web-based services where needed. Lastly, this and other studies demonstrate that utilizing the experiences and expertise of parents in the study design effectively creates a program that matches the targeted population's needs. ${ }^{11}$ Therefore, hospital or community-based programs desiring to develop a mindfulness-based program should consider involving local parents in the design and implementation.

This study highlights the need for and benefits of providing parents of children with special needs, especially those with ADHD and autism, with an accessible mindfulness-based program to manage excessive stress. Web-based platforms provide a sustainable approach to family-based interventions. It allows more parents to participate around the world, minimizes the time and expenses of traveling to a facility-based group, and may be a viable option for a busy parent's lifestyle. Research continues to reveal the negative implications of excessive stress on the health, well-being, and functioning within the family. As the number of children diagnosed with neurodevelopmental disorders increases, utilizing innovative programs offering tools to manage the excessive stress experienced by families who have children with complex needs should be considered.

\section{REFERENCES}

1. Sharpley CF, Bitsika V, Efremidis B. Influence of gender, parental health, and perceived expertise of assistance upon stress, anxiety, and depression among parents of children with autism. Journal of Intellectual and Developmental Disability. 1997;22(1):19-28. doi:10.1080/13668259700033261.

2. Hayes SA, Watson SL. The impact of parenting stress: A meta-analysis of studies comparing the experience of parenting stress in parents of children with and without autism spectrum disorder. Journal of Autism and Developmental Disorders. 2013;43(3):629-642. 
3. Ferraioli SJ, Harris SL. Comparative effects of mindfulness and skills-based parent training programs for parents of children with autism: Feasibility and preliminary outcome data. Mindfulness. 2013;4(2):89-10. doi:10.1007/s12671-0120099-0.

4. Heath CL, Curtis DF, Fan W, McPherson R. The association between parenting stress, parenting self-efficacy, and the clinical significance of child ADHD symptom change following behavior therapy. Child Psychiatry Hum Dev. 2015;46:11829.[PMID: 24668566]

5. Behbahani M, Zargar F, Assarian F, Akbari H. Effects of mindful parenting training on clinical symptoms in children with attention deficit hyperactivity disorder and parenting stress: Randomized controlled trial. Iran J Med Sci. 2018;43(6):596604.

6. Krakovich TM, McGrew JH, Yu Y, Ruble LA. Stress in parents of children with autism spectrum disorder: An exploration of demands and resources. Journal of Autism and Developmental Disorders. 2016;46(6):2042-2053. doi:10.1007/s10803016-2728-2.

7. Garcia AS, Ren L, Esteraich JM, Raikes HH. Influence of child behavioral problems and parenting stress on parent-child conflict among low-income families: The moderating role of maternal nativity. Merrill-Palmer Quarterly: Journal of Developmental Psychology. 2017;63(3):311-339.

8. Costa AP, Steffgen G, Ferring D. Research Paper: Contributors to well-being and stress in parents of children with autism spectrum disorder. Research in Autism Spectrum Disorders. 2017;37:61-72. doi:10.1016/j.rasd.2017.01.007.

9. Hsiao Y-J. Pathways to mental health-related quality of life for parents of children with autism spectrum disorder: Roles of parental stress, children's performance, medical support, and neighbor support. Research in Autism Spectrum Disorders. 2016;23:122-130. doi:10.1016/j.rasd.2015.10.008.

10. Campbell K, Thoburn JW, Leonard HD. The mediating effects of stress on the relationship between mindfulness and parental responsiveness. Couple and Family Psychology: Research and Practice. 2017;6(1):48-59. doi:10.1037/cfp0000075.

11. Bazzano A, Wolfe C, Zylowska L, Wang S, Schuster E, Barrett C, et al. Mindfulness-based stress reduction (MBSR) for parents and caregivers of individuals with developmental disabilities: A community-based approach. Journal of Child and Family Studies. 2015; 24(2):298-308.

12. Shonkoff JP, Garner AS, Committee on Psychosocial Aspects of Child and Family Health, Committee on Early Childhood, Adoption, and Dependent Care, Section on Developmental and Behavioral Pediatrics. The lifelong effects of early childhood adversity and toxic stress. Pediatrics. 2012;129(1):e232-e246. doi:10.1542/peds.2011-2663.

13. Yorke I, White P, Weston A, Rafla M, Charman T, Simonoff E. The Association between emotional and behavioral problems in children with autism spectrum disorder and psychological distress in their parents: A systematic review and meta-analysis. Journal of Autism and Developmental Disorders. 2018;48(10):3393-3415. https://link.springer.com/article/10.1007\%2Fs10803-018-3605-y. Accessed January 29, 2019.

14. Dykens EM, Fisher MH, Taylor JL, Lambert W, Miodrag N. Reducing distress in mothers of children with autism and other disabilities: A randomized trial. Pediatrics. 2014;134(2):454-463.

15. Osborne LA, McHugh L, Saunders J, Reed P. Parenting stress reduces the effectiveness of early teaching interventions for autistic spectrum disorders. Journal of Autism and Developmental Disorders. 2008;38(6):1092-1103.

16. Zhou T, Yi C. Parenting styles and parenting perspectives on how their own emotions affect the functioning of their children with autism spectrum disorders. Fam Process. 2014;53:67-79.

17. Cachia RL, Anderson A, Moore DW. Mindfulness, stress and well-being in parents of children with autism spectrum disorder: A systematic review. Journal of Child and Family Studies. 2016;25(1):1-14. doi:10.1007/s10826-015-0193-8.

18. Neece CL. Mindfulness-based stress reduction for parents of young children with developmental delays: Implications for parental mental health and child behavior problems. Journal of Applied Research in Intellectual Disabilities. 2014;27(2):174-186. doi:10.1111/jar.12064.

19. Singh NN, Lancioni GE, Karazsia BT, Chan J, Winston AS. Effectiveness of caregiver training in mindfulness-based positive behavior supports (MBPBS) vs. training- as-usual (TAU): A randomized controlled trial. Frontiers in Psychology. 2016;7(1549):1-13. doi:10.3389/fpsyg.2016.01549.

20. Benn R, Roeser RW, Arel S, Akiva T. Mindfulness training effects for parents and educators of children with special needs. Developmental Psychology. 2012;48(5):1476-1487

21. Shapero BG, Greenberg J, Mischoulon D, Pedrelli P, Meade K, Lazar SW. Mindfulness-based cognitive therapy improves cognitive functioning and flexibility among individuals with elevated depressive symptoms. Mindfulness. 2018;9(5):1457.

22. Lewallen A, Neece C. Improved social skills in children with developmental delays after parent participation in MBSR: The role of parent-child relational factors. Journal of Child \& Family Studies. 2015;24(10):3117-3129. doi:10.1007/s10826-0150116-8. Accessed January 9, 2019.

23. Neff KD, Faso DJ. Self-compassion and well-being in parents of children with Autism. Mindfulness. 2015;6(4):938-947. doi:10.1007/s12671-014-0359-2. 
24. Baum F, MacDougall C, Smith D. Participatory action research. Journal of Epidemiology \& Community Health. 2006;60(10):854-857. doi:10.1136/jech.2004.028662

25. Kabat-Zinn J. Full Catastrophe Living (Revised Edition): Using the Wisdom of Your Body and Mind to Face Stress, Pain, and Illness. New York: Bantam Books, 2013.

26. Stahl B, Goldstein E. A Mindfulness-Based Stress Reduction Workbook. Oakland, CA: New Harbinger Publications, 2010.

27. Lovibond SH, Lovibond PF. Manual for the Depression Anxiety Stress Scales (DASS). $2^{\text {nd }}$ ed. Sydney, Australia: University of New South Wales. 1995. http://www2.psy.unsw.edu.au/dass. Accessed March 14, 2018.

28. Cohen S. Perceived Stress Scale. Mind Garden. https://www.mindgarden.com/132-perceived-stress-scale. Accessed June 11, 2018.

29. Baer RA, Smith GT, Lykins E, Button D, Krietemeyer J, Sauer S, et al. FFMQ-15 items and scoring information. Research Gate, 2016. https://www.researchgate.net/publication/303709635_FFMQ-15_items_and_scoring_information. Accessed March 12, 2018.

30. Crawford J, Cayley C, Lovibond PF, Wilson PH, Hartley C. Percentile norms and accompanying interval estimates from an Australian general adult population sample for self-report mood scales (BAI, BDI, CRSD, CES-D, DASS, DASS-21, STAIX, STAI-Y, SRDS, and SRAS). Australian Psychologist. 2011;46(1):3-14. doi:10.1111/j.1742-9544.2010.00003.x.

31. Andreou E, Alexopoulos EC, Lionis C, Varvogli L, Gnardellis C, Chrousos GP, et al. Perceived Stress Scale: Reliability and validity study in Greece. Int J Environ Res Public Health. 2011;8(8):3287-98.

32. Meppelink R, de Bruin El, Wanders-Mulder FH, Vennik CJ, Bögels SM. Mindful parenting training in child psychiatric settings: Heightened parental mindfulness reduces parent's and children's psychopathology. Mindfulness. 2016;7(3):680689. doi:10.1007/s12671-016-0504-1.

33. Gu J, Strauss C, Crane C, Barnhofer T, Karl A, Cavanagh K, et al. Examining the factor structure of the 39-item and 15item versions of the Five Facet Mindfulness Questionnaire before and after mindfulness-based cognitive therapy for people with recurrent depression. Psychol Assess. 2016;28(7):791-802. doi:10.1037/pas0000263

34. Inductive or deductive? Two different approaches. Saylor Academy. https://www.saylordotorg.github.io/text_principles-ofsociological-inquiry-qualitative-and-quantitative-methods/s05-03-inductive-or-deductive-two-dif.html. Accessed September 23, 2019.

35. Salazar F, Baird G, Chandler S, Tseng E, O'sullivan T, Howlin P, et al. Co-occurring psychiatric disorders in preschool and elementary school-aged children with Autism Spectrum Disorder. Journal of Autism \& Developmental Disorders. 2015;45(8):2283-2294. doi:10.1007/s10803-015-2361-5.

36. Jackson AC, Liang RP, Frydenberg E, Higgins RO, Murphy BM. Parent education programmes for special health care needs children: A systematic review. Journal of Clinical Nursing. 2016;25(11-12):1528-1547. doi:10.1111/jocn.13178.

37. Henry BW, Ames LJ, Block DE, Vozenilek JA. Experienced practitioners' views on interpersonal skills in telehealth delivery. The Internet Journal of Allied Health Sciences and Practice. 2018 Apr 05;16(2), Article 2.

38. Gross D, Grady J. Group-based parent training for preventing mental health disorders in children. Issues in Mental Health Nursing. 2002;23: 367-383. 